\title{
Black Hole Entropy in Induced Gravity and Information Loss
}

\author{
D.V. Fursaev ${ }^{\mathrm{a}}$

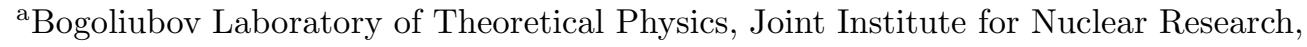 \\ 141980 Dubna, Russia
}

\begin{abstract}
The basic assumption of the induced gravity approach is that Einstein theory is an effective, low energy-form of a quantum theory of constituents. In this approach the Bekenstein-Hawking entropy $S^{B H}$ of a black hole can be interpreted as a measure of the loss of information about constituents inside the black hole horizon. To be more exact, $S^{B H}$ is determined by quantum correlations between "observable" and "non-observable" states with positive and negative energy $\mathcal{E}$, respectively. It is important that for non-minimally coupled constituents $\mathcal{E}$ differs from the canonical Hamiltonian $\mathcal{H}$. This explains why previous definitions of the entanglement entropy in terms of $\mathcal{H}$ failed to reproduce $S^{B H}$.
\end{abstract}

The idea of induced gravity has been formulated first by A.D.Sakharov [1]. Its basic assumption is that the dynamical equations for the gravitational field $g_{\mu \nu}$ are related to properties of the physical vacuum which has a microscopic structure like a usual medium. A suitable example for the comparison is a crystal lattice. The metric $g_{\mu \nu}$ plays a role similar to a macroscopic displacement in the crystal and gravitons are collective excitations of the microscopic constituents of the vacuum analogous to phonons. In the crystal, a deformation results in the change of the energy expressed in terms of displacements. In a similar way, the physical vacuum responses to variations of $g_{\mu \nu}$ by changing its energy due to polarization effects. These quantum effects can be described by the effective action $\Gamma\left[g_{\mu \nu}\right]$ for the metric

$e^{i \Gamma\left[g_{\mu \nu}\right]}=\int[D \Phi] e^{i I\left[\Phi, g_{\mu \nu}\right]}$.

The notation $\Phi$ stands for the constituent fields, and $I\left[\Phi, g_{\mu \nu}\right]$ denotes their classical action. Sakharov's idea is based on the observation that one of leading contributions to $\Gamma\left[g_{\mu \nu}\right]$ has a form of the Einstein action.

Why the idea of induced gravity has something to do with the problem of the black hole entropy? The Bekenstein-Hawking entropy

$S^{B H}=\frac{1}{4 G} \mathcal{A}$,

where $\mathcal{A}$ is the area of the horizon and $G$ is the
Newton constant, appears under analysis of the laws of black hole mechanics in classical Einstein theory. The induced gravity is endowed with a microscopic structure, constituents, which can be related naturally to degrees of freedom responsible for $S^{B H}$. As was first pointed out in [2], the mechanism which makes explanation of $S^{B H}$ in induced gravity possible is the quantum entanglement [3]. The constituents in the black hole exterior are in the mixed state due to the presence of the horizon. This state is characterized by the entropy $S$ of entanglement. In a local field theory correlations between external and internal regions are restricted by the horizon surface, and so $S=\mathcal{A} / l^{2}$. The constant $l$ is the same ultraviolet cutoff which is required to make effective action (11) finite 沟. Because the induced Newton constant $G$ in $\Gamma\left[g_{\mu \nu}\right]$ is proportional to $l^{2}$ entropy $S$ is comparable to $S^{B H}$.

To understand whether $S^{B H}$ is a "purebred" entanglement entropy one has to avoid the cutoff procedure and consider a theory which is free from the divergences. The Bekenstein-Hawking entropy is a pure low-energy quantity. It was suggested in 5 that the mechanism of generation of $S^{B H}$ is universal for the theories where gravity is completely induced by quantum effects, as in Sakharov's approach or in string theory, and has ultraviolet finite induced Newton constant $G$. The last requirement makes the theory selfconsistent and enables one to carry out the com- 
putations. The universality means that $S^{B H}$ may be understood without knowing details of quantum gravity theory [5] (see also [6] for a different realization of this idea).

The models which obey these requirements were constructed in [5,7 10]. The constituents are chosen there as non-interacting scalar, spinor and vector fields. They have different masses comparable to the Planck mass $m_{P l}$. The masses and other parameters obey certain constraints which provide cancellation of the leading (or all 10]) divergences in effective gravitational action (11). In the low-energy limit when curvature $R$ of the background is small as compared to $m_{P l}^{2}$

$\Gamma\left[g_{\mu \nu}\right] \simeq \frac{1}{16 \pi G} \int \sqrt{-g} d^{D} x(R-2 \Lambda) \quad$.

The induced Newton constant $G$ and the cosmological constant $\Lambda$ are the functions of parameters of the constituents and can be computed. In four dimensions one imposes further restrictions to eliminate the cosmological term. To study charged black holes one has to generalize Sakharov's proposal and consider the induced Einstein-Maxwell theory (see the details in [10]).

All above models include bosonic constituents (scalars or vectors) with a positive non-minimal coupling to the background curvature. The presence of such couplings is unavoidable if one wants to eliminate the divergences in $G$. The Bekenstein-Hawking entropy of a non-extremal stationary black hole has the following form [5,7 10]

$$
\begin{aligned}
& S^{B H}=\frac{1}{4 G} \mathcal{A}=S-\mathcal{Q}, \\
& S=-\operatorname{Tr} \rho \ln \rho \quad, \quad \rho=\mathcal{N} e^{-\beta_{H} \mathcal{H}} .
\end{aligned}
$$

Here $S$ is the thermal entropy of the constituents propagating in Planck region near the black hole horizon and $\beta_{H}^{-1}=\frac{\kappa}{2 \pi}$ is the Hawking temperature. The operator $\mathcal{H}$ is the canonical Hamiltonian which generates translations along the Killing vector field $\xi\left(\xi^{2}=0\right.$ on the horizon). Quantity $\mathcal{Q}$ is the vacuum average of an operator which has the form of the integral over the horizon $\Sigma$. $\mathcal{Q}$ is determined only by the nonminimal couplings of the constituents and it can be interpreted as a Noether charge on $\Sigma$ defined with respect to field $\xi$ [7, 11]. Formula (41) is absolutely universal in a sense it depends neither on the choice of constituent species nor on a type of a black hole. The entropy $S$ is always positive and divergent and so does $\mathcal{Q}$ in the considered models. Subtracting $\mathcal{Q}$ in (4) compensates divergences of $S$ rendering $S^{B H}$ finite. Thus, nonminimal couplings provide both finiteness of the effective action and of $S^{B H}$.

The physical meaning of $\mathcal{Q}$ becomes evident if we analyze the classical energy of the nonminimally coupled constituents defined on a space-like hypersurface $\Sigma_{t}$ which crosses the bifurcation surface $\Sigma$

$\mathcal{E}=\int_{\Sigma_{t}} T_{\mu \nu} \xi^{\mu} d \Sigma^{\nu}$

Here $T_{\mu \nu}$ is the stress-energy tensor and $d \Sigma^{\mu}$ is the future-directed vector of the volume element on $\Sigma_{t}$. For the fields in black hole exterior [11]

$\mathcal{E}=\mathcal{H}-\frac{\kappa}{2 \pi} \mathcal{Q}$,

where $\mathcal{H}$ is the canonical Hamiltonian. The difference between the two energies is a total divergence which results in boundary terms. $\mathcal{Q}$ is the contribution from the horizon $\Sigma$ which is an internal boundary of $\Sigma_{t}$. The energies $\mathcal{H}$ and $\mathcal{E}$ play different roles: $\mathcal{H}$ is the generator of canonical transformations along $\xi$, while $\mathcal{E}$ is the observable energy which is related to thermodynamical properties of a black hole. Indeed, there is the classical variational formula 11

$\delta M=\frac{\kappa}{2 \pi} \delta S^{B H}+\delta \mathcal{E}$

which relates the change of the black hole mass at infinity $\delta M$ to the change of the entropy $\delta S^{B H}$ under a small perturbation of the energy $\delta \mathcal{E}$ of matter fields.

Suppose now that the total mass $M$ is fixed. Then, the change of $S^{B H}$ is completely determined by change of the energy $\mathcal{E}$ in the black hole exterior. In classical processes the matter is falling down in the black hole. It results in decreasing of the energy $\delta \mathcal{E}<0$ and increasing of the black hole entropy $S^{B H}$. In quantum theory $S^{B H}$ may decrease due to the Hawking effect. In 
such a process $\delta \mathcal{E}>0$, and a black hole absorbs a negative energy.

We can now make two observations. First, according to (5), changes of $S$ are related to changes of the canonical energy $\mathcal{H}$, and because $\mathcal{H}$ and $\mathcal{E}$ are different, the two entropies, $S^{B H}$ and $S$, should be different as well. Second, the conclusion whether a physical process results in a loss of the information inside the black hole can be made by analyzing the sign of the energy change $\delta \mathcal{E}$. Note that using for this purpose the sign of the canonical energy would result in a mistake. For instance, if $\mathcal{Q}>0$, there may be classical processes where $\mathcal{H}$ and $S^{B H}$ increase simultaneously, see (7), (8).

It is known that the thermal entropy $S$, defined in (5), can be interpreted as an entanglement entropy [12]. This is true when one is interested in correlations (entanglement) of states characterized by the certain canonical energy $\mathcal{H}$. However, to describe a real information loss one has to study correlations between observable and nonobservable states which have positive and negative energy $\mathcal{E}$, respectively.

Consider a pair creation process near the black hole horizon. The antiparticle of a pair carries a negative energy. It tunnels through the horizon and becomes non-observable. As a consequence, the observed particle is in a mixed state. The entropy which describes the corresponding information loss can be defined by the Shannon formula

$S^{I}=-\sum_{\mathcal{E}} p(\mathcal{E}) \ln p(\mathcal{E})$

Here $p(\mathcal{E})$ is the probability to observe a particle with the certain energy $\mathcal{E}$. Obviously, $p(\mathcal{E})$ coincides with the probability for the antiparticle with energy $-\mathcal{E}$ to tunnel through the horizon. This process can be also described in another form. Consider a Kruskal extension of the black hole space-time near the horizon. Then the surface $\Sigma_{t}$ consists of the two parts, $\Sigma_{t}^{R}$ (right) and $\Sigma_{t}^{L}$ (left), which are separated by the bifurcation surface $\Sigma$. The creation of a pair is equivalent to quantum tunneling of a particle moving backward in time from the left region to the right. The corresponding amplitude is the evolution operator from $\Sigma_{t}^{L}$ to $\Sigma_{t}^{R}$ in Euclidean time $\tau$. In the Hartle-Hawking vacuum $\tau=\beta_{H} / 2$.

How to describe the tunneling probability? First, note that the energy has a form of the Hamiltonian modified by a "perturbation" $\mathcal{Q}$. Such a modified Hamiltonian may be considered as an operator which generates evolution of states having certain energies. Then, the operator

$T=e^{-\frac{\beta_{H}}{2} \mathcal{E}}$

corresponds to the tunneling transition. Second, because a particle on $\Sigma_{t}^{L}$ is moving backward in time, $\mathcal{E}$ should be defined by (6) with the pastoriented Killing field $\xi$. It means that relation (7) should be written now as

$\mathcal{E}=\mathcal{H}+\frac{\kappa}{2 \pi} \mathcal{Q}$,

if one leaves for $\mathcal{Q}$ the former expression. (So $\mathcal{Q}$ is positive for positive non-minimal couplings.) The same relation is true for the energy and Hamiltonian of an antiparticle tunneling from $\Sigma_{R}^{t}$ to $\Sigma_{L}^{t}$.

The probability $p(\mathcal{E})$ which involves transitions from all states in one region to a state with the energy $\mathcal{E}$ in the other region is

$p(\mathcal{E})=\mathcal{N}\left\langle\mathcal{E}\left|e^{-\left(\beta_{H} \mathcal{H}+\mathcal{Q}\right)}\right| \mathcal{E}\right\rangle$.

Here $\mathcal{Q}$ is the operator which corresponds to the Noether charge. We now have to calculate $S^{I}$ by using (9) and (12). To this aim it is convenient to introduce a free energy $F^{I}(\beta)$

$e^{-\beta F^{I}(\beta)}=\operatorname{Tr} e^{-\beta \mathcal{E}}$,

which makes it possible to rewrite $S^{I}$ as

$S^{I}=\left.\beta^{2} \partial_{\beta} F^{I}(\beta)\right|_{\beta=\beta_{H}}$.

Our last step is to calculate $F^{I}(\beta)$. This can be done by using our interpretation of $\mathcal{Q}$ as a perturbation. Then in the linear order

$$
\begin{aligned}
& F^{I}(\beta)=F(\beta)+\beta_{H}^{-1}\langle\mathcal{Q}\rangle_{\beta}, \\
& e^{-\beta F(\beta)}=\operatorname{Tr} e^{-\beta \mathcal{H}}, \\
& \langle\mathcal{Q}\rangle_{\beta}=\operatorname{Tr}\left(\mathcal{Q} e^{-\beta \mathcal{H}}\right) e^{\beta F(\beta)} .
\end{aligned}
$$

Here $F(\beta)$ is the standard free energy for a canonical ensemble at the temperature $\beta^{-1}$. $\langle\mathcal{Q}\rangle_{\beta}$ is the thermal average of the Noether charge in 
this canonical ensemble. As has been argued in [7], this average is determined by contributions of particles with negligibly small canonical energies $\omega$ (the soft modes). The density $n_{\omega}$ of Bose particles at the temperature $\beta^{-1}$ is singular and proportional to the temperature, $n_{\omega} \simeq(\beta \omega)^{-1}$. Thus,

$\langle\mathcal{Q}\rangle_{\beta}=\frac{\beta_{H}}{\beta}\langle\mathcal{Q}\rangle_{\beta_{H}}$

where $\langle\mathcal{Q}\rangle_{\beta_{H}} \equiv \mathcal{Q}$ is the average in the HartleHawking vacuum. By using (14), (15) and (16) one finds

$S^{I}=\left.\beta^{2} \partial_{\beta} F(\beta)\right|_{\beta=\beta_{H}}-\langle\mathcal{Q}\rangle_{\beta_{H}}=S-Q$.

Here we used the fact that $F(\beta)$ determines thermal entropy (5). Equation (19) proves our interpretation of the Bekenstein-Hawking entropy in induced gravity (4) as the entropy related to the information loss.

We conclude with the several remarks.

1) In induced gravity $S^{B H}$ counts only the states of the constituents located near the horizon, the "surface states". Thus, as was argued in [13], $S^{B H}$ should not be related to internal states of the black hole which cannot influence outside.

2) Our arguments are applied to the entropy of Rindler observers and give for this quantity the finite value $(4 G)^{-1}$ per unit surface.

3 ) It was suggested in [7] to relate $S^{B H}$ to degeneracy of the spectrum of the mass of the black hole $M_{H}$ measured at the horizon. If mass at infinity $M$ is fixed, change of $M_{H}$ coincides with the energy of an antiparticle absorbed by a black hole in the course of the pair creation process. According to arguments above, this energy has to be determined by (11) and it is the operator that appears in density matrix (12). Thus, our present interpretation of $S^{B H}$ agrees with [7].

4) Density matrix (12) differs from the canonical density matrix by insertion of the operator $\mathcal{Q}$ on $\Sigma$. This insertion changes only the distribution of the soft modes. The divergence of the thermal entropy $S$ in induced gravity can be related to the degeneracy of states with respect to soft modes [7] because adding a soft mode does not change the canonical energy of the state. The insertion of $\mathcal{Q}$ in (12) removes this degeneracy. At the same time averages of operators located outside $\Sigma$ do not depend on this insertion.

Acknowledgements: This work is supported in part by the RFBR grant N 99-02-18146.

\section{REFERENCES}

1. A.D. Sakharov, Sov. Phys. Doklady, 12 (1968) 1040.

2. T. Jacobson, Black Hole Entropy in Induced Gravity, gr-qc/9404039.

3. L. Bombelli, R.K. Koul, J. Lee, and R. Sorkin, Phys. Rev. D34 (1986) 373; M. Srednicki, Phys. Rev. Lett. 71 (1993) 666; V. Frolov and I. Novikov, Phys. Rev. D48 (1993) 4545.

4. L. Susskind and J. Uglum, Phys. Rev. D50 (1994) 2700, C. Callan and F. Wilczek, Phys. Lett. B333 (1994) 55.

5. V.P. Frolov, D.V. Fursaev and A.I. Zelnikov, Nucl. Phys. B486 (1997) 339.

6. S. Carlip, Class. Quantum Grav. 16 (1999) 3327, S. Solodukhin, Phys. Lett. B454 (1999) 213.

7. V.P. Frolov and D.V. Fursaev, Phys. Rev. D56 (1997) 2212.

8. V.P. Frolov and D.V. Fursaev, Phys. Rev. D58 (1998) 124009.

9. V.P. Frolov and D.V. Fursaev, Statistical Mechanics on Axially-symmetric Space-time with the Killing Horizon and Entropy of Rotating Black Holes in Induced Gravity, grqc/9907046.

10. V.P. Frolov and D.V. Fursaev, Statistical Mechanics of Charged Black Holes in Induced Einstein-Maxwell Gravity, hep-th/9910006.

11. D.V. Fursaev, Phys. Rev. D59 (1999) 064020.

12. W. Israel, Phys. Lett. 57A (1976) 107, T. Jacobson, Phys. Rev. D50 (1994) 6031.

13. R.D. Sorkin, Statistical Mechanics of Black Hole Thermodynamics, gr-qc/9705006; T. Jacobson, On the Nature of Black Hole Entropy, gr-qc/9908031. 